\title{
Macrofauna associada às galerias de Neoteredo reynei (Bartsch, 1920) (Mollusca: Bivalvia) em troncos de Rhizophora mangle Linnaeus durante o período menos chuvoso, em manguezal de São Caetano de Odivelas, Pará (costa norte do Brasil) \\ Macrofauna associated with galleries of Neoteredo reynei (Bartsch, 1920) (Mollusca: Bivalvia) in Rhizophora mangle Linnaeus trunks during less rainy season in mangrove of São Caetano de Odivelas, Pará (North Coast of Brazil)
}

Resumo: Este estudo teve como objetivo caracterizar a macrofauna associada às galerias perfuradas por Neoteredo reynei em toras de Rhizophora mangle em uma área de manguezal do estuário do rio Mojuim (São Caetano de Odivelas, Pará). As coletas foram realizadas em três ocasiões do período menos chuvoso (junho, julho e agosto de 2004), na zona de médio-litoral em três sítios de amostragem. Em cada ocasião, foi retirada, de cada sítio, uma tora de $R$. mangle em estado inicial de decomposição. Paralelamente à coleta biológica, foram registrados a temperatura do ar, o $\mathrm{pH}$, a salinidade e a temperatura da água. Neoteredo reynei foi a única espécie de Teredinidae presente nas amostras. A macrofauna associada às suas galerias foi composta por um total de 452 indivíduos, distribuídos em 31 táxons pertencentes aos filos: Nemertea (um táxon), Mollusca (três táxons), Annelida (seis táxons) e Arthropoda (21 táxons). A composição da fauna registrada foi tipicamente estuarina, com exceção de alguns grupos terrestres. Os descritores biológicos (abundância, riqueza, diversidade e equitatividade) aumentaram seus valores ao longo dos meses, como provável reflexo do aumento da salinidade no estuário. A diversidade da macrofauna registrada no interior das toras reflete a importância deste nicho para os organismos e, consequentemente, para o funcionamento do ecossistema manguezal.

Palavras-chave: Bentos. Costa norte. Estuário. Teredinidae. Fauna associada.

\begin{abstract}
This study aims to characterize the benthic macrofauna associated with galleries made by Neoteredo reynei in Rhizophora mangle mangroves of the river Mojuim estuary (São Caetano de Odivelas, Pará). Samples were taken during the less rainy period of 2004 (June, July and August) at three sites in the intertidal zone. At every occasion a log of $R$. mangle in the initial stages of decomposition was removed from each area. During the biological sampling air temperature, $\mathrm{pH}$, salinity and temperature of the water were recorded. Neoteredo reynei was the only Teredinidae (boring mussel) species observed. The macrofauna was composed of 452 individuals, distributed over 31 taxa belonging to the phyla Nemertea (one tax), Mollusca (three taxa), Annelida (six taxa) and Arthropoda (21 taxa). The recorded fauna was typically estuarine, whit the exception of some terrestrial groups. Abundance, richness, diversity and uniformity increased in the course of the dry season, probably reflecting the observed increase of water salinity. The high diversity of the macrofauna recorded in this study reinforces the importance of teredo galleries as a habitat for estuarine organisms and consequently for the functioning of the mangrove ecosystem.
\end{abstract}

Keywords: Benthos. North coast. Estuary. Teredinidae. Associated fauna.

l Universidade Federal do Pará. Faculdade de Oceanografia. Instituto de Geociências. Belém, Pará, Brasil (daiane.aviz@gmail.com). " Universidade Federal Rural da Amazônia. Departamento de Ciências Aquáticas. Belém, Pará, Brasil (clara@amazon.com.br).

III Secretaria de Educação do Estado do Pará. São Caetano de Odivelas, Pará, Brasil (fernandespat@yahoo.com.br).

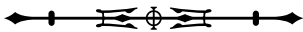




\section{INTRODUÇÃO}

As associações animais mono- e poliespecíficas são comuns em comunidades bentônicas do entre-marés (Levinton, 2001). Grande parte dos organismos sésseis pode se associar a uma variedade de pequenos invertebrados que vivem como endo- ou epibiontes. Colônias de esponjas (Frith, 1976), cnidários (Young, 1986) e moluscos (Thiel \& Ullrich, 2002) são microhabitats amplamente utilizados por muitas espécies. Outros animais, embora não forneçam sua superfície e cavidades corporais como moradia, são capazes de construir estruturas físicas estáveis, adequadas ao estabelecimento e sobrevivência de uma diversificada biota. Entre estas estruturas, podem-se citar os tubos de poliquetas (Sepúlveda et al., 2003), as galerias de talassanídeos (Branch \& Pringle, 1987) e de teredinídeos (Turner, 1966).

Os teredos, moluscos bivalves marinhos pertencentes à família Teredinidae, se fixam na madeira no final de sua fase larval e, à medida que crescem, constroem longas galerias revestidas de calcário (Muller \& Lana, 2004). É de grande importância a atuação desses animais na reciclagem da matéria orgânica de origem vegetal, notadamente em áreas de manguezal, que contribuem substancialmente para o enriquecimento das águas estuarinas por meio da exportação de detritos. Os Teredinidae aceleram a destruição da madeira lançada pelos rios ao mar, principalmente por aumentar a superfície em exposição, propiciando que um maior número de bactérias se deposite no local (Turner, 1966; Rimmer et al., 1983).

Em manguezais, onde o sedimento apresenta grande instabilidade física, alto conteúdo de sal, baixos teores de oxigênio e a ocorrência de substrato consolidado é limitada (Camargo, 1986; Duke et al., 1998), as galerias construídas pelos teredos no interior de troncos caídos são habitats ideais para diversos grupos animais, sobretudo para pequenos invertebrados bentônicos (Turner \& Johnson, 1971). Poucos são os estudos sobre a fauna presente nestas galerias, os trabalhos já realizados no Brasil estão basicamente restritos à costa do Pará e do Ceará, sob forma de monografias, teses e resumos de congressos científicos, merecendo destaque a Tese de Doutorado de Clara Pantoja Ferreira, defendida no ano de 1989, cujo título é "Manguezais do Estado do Pará: Endofauna de galerias perfuradas por teredos em toras de Rhizophora", que investigou tal fauna em alguns municípios paraenses; e o estudo de Santos et al. (2007), realizado em manguezal do estado do Ceará.

Tendo em vista a relevância ecológica e a falta de informações sobre os organismos que vivem neste habitat, o presente trabalho teve por objetivo caracterizar a macrofauna associada às galerias perfuradas por teredos em toras de Rhizophora mangle L. em uma área de manguezal do município de São Caetano de Odivelas, Pará, costa norte do Brasil.

\section{MATERIAL E MÉTODOS}

\section{CARACTERIZAÇÃO DA REGIÃO}

A área de estudo está situada na faixa litorânea do município de São Caetano de Odivelas, às margens do rio Mojuim, cujo estuário é delimitado pelos paralelos: $00^{\circ} 35^{\prime} 00^{\prime \prime} \mathrm{Se}$ $47^{\circ} 03^{\prime} 45^{\prime \prime} \mathrm{W}, 00^{\circ} 52^{\prime} 30^{\prime \prime} \mathrm{S}$ e $48^{\circ} 05^{\prime} 00^{\prime \prime} \mathrm{W}$. A região é dominada por meso-marés semidiurnas, com amplitudes entre 2 e 4 m (Prost et al., 2001).

A região apresenta clima equatorial amazônico do tipo Am (classificação de Köppen), com temperaturas elevadas (média de $27^{\circ} \mathrm{C}$ ), pequena amplitude térmica e precipitações médias anuais de 2.500 mm (Martorano, 1993). A variação temporal da precipitação é caracterizada por um período chuvoso, que, em geral, compreende os meses de dezembro a maio, com média de $1.657 \mathrm{~mm}$, e por um período seco ou menos chuvoso, de junho a novembro, com média de 487 mm (Moraes et al., 2005).

\section{TRABALHO DE CAMPO E LABORATÓRIO}

As coletas foram realizadas no período menos chuvoso de 2004, nos meses de junho, julho e agosto. A área de manguezal estudada está localizada na margem continental do rio Mojuim, entre o Porto São Pedro (a jusante), um dos principais da cidade de São Caetano de Odivelas, e o 
igarapé Jutaí (a montante), canal de maré do rio Mojuim. Nesta área, foram estabelecidos três sítios de coleta, o primeiro (P1) situado a cerca de $100 \mathrm{~m}$ do Porto São Pedro, o segundo (P2) e o terceiro (P3) situados a cerca de 150 e $300 \mathrm{~m}$, respectivamente, do primeiro sítio e a cerca de 200 e 50 m, respectivamente, do igarapé Jutaí.

Em cada sítio, na zona de médio-litoral superior, em áreas vegetadas, estabeleceu-se um raio de amostragem com aproximadamente $10 \mathrm{~m}^{2}$. Em cada mês, coletou-se aleatoriamente, em cada sítio, uma tora de Rhizophora mangle em estado inicial de decomposição, com aproximadamente $60 \mathrm{~cm}$ de comprimento e $30 \mathrm{~cm}$ de diâmetro. No final do período de amostragem, foi coletado um total de nove toras de $R$. mangle. Cada tora foi acondicionada em saco plástico devidamente etiquetado e levada ao laboratório para triagem. Paralelamente à coleta biológica, foram registrados, junto à margem do rio Mojuim, em área não vegetada, os valores da temperatura da água e do ar, assim como a salinidade e o pH da água superficial.

Foram levantados, ainda, dados de precipitação pluviométrica acumulada para os meses de amostragem junto ao Instituto Nacional de Meteorologia (INMET), da estação meteorológica de Belém, Pará, a mais próxima da região de estudo, distando cerca de $95 \mathrm{~km}$.

Em laboratório, procedeu-se a observação minuciosa das toras para a retirada dos organismos da sua superfície e sob a casca. Em seguida, as toras foram mergulhadas em solução de cloreto de magnésio a 10\%, para relaxar e anestesiar os organismos. Após 15 minutos, elas foram lavadas em água corrente sobre um sistema de peneiras de malhas de diferentes diâmetros (1,0 e $0,5 \mathrm{~mm}$ ). Posteriormente, as toras foram fragmentadas longitudinalmente, seus pedaços foram lavados sobre o mesmo sistema de peneira e triados com auxílio de um microscópio estereoscópio. Os organismos encontrados na superfície das toras ou sob a casca não foram considerados na análise, apenas se contabilizou aqueles removidos do interior e em volta das galerias de teredos, principalmente do córtex e regiões mais basais. Os invertebrados foram identificados ao menor nível taxonômico possível, contados e conservados em álcool etílico a 70\%.

Em todos os meses, a amostragem em cada sítio foi realizada em dias alternados, dado o esforço para a fragmentação e triagem dos organismos das toras, que eram iniciadas, no máximo, uma hora e meia após a coleta.

\section{ANÁLISE DOS DADOS}

Foram determinados para cada amostra a abundância (número total de indivíduos), riqueza (número total de táxons), diversidade (índice H' de Shannon-Wiener, utilizando log e) e equitatividade (índice J de Pielou). Para a comparação dos descritores biológicos, entre as ocasiões de coleta, foi utilizado o teste "t student" não pareado. Anteriormente à realização das análises, os dados foram testados quanto à normalidade e à homogeneidade das variâncias. A significância estatística foi considerada quando $p<0,05$.

\section{RESULTADOS E DISCUSSÃO}

\section{PARÂMETROS AMBIENTAIS}

As temperaturas do ar e da água estiveram sempre acima de $26^{\circ} \mathrm{C}$, em geral, com maiores valores nos meses de julho e agosto e nos sítios P2 e P3 (Tabela 1). A salinidade da água apresentou marcada variação temporal, com um crescente aumento no decorrer dos meses, acompanhando os padrões de precipitação pluviométrica na região (Tabela 1). Entre sítios, maiores valores de salinidade foram observados em P3, e menores, em $\mathrm{P} 1$. Os valores de $\mathrm{pH}$ se mantiveram sempre próximos a sete (Tabela 1), sem padrões temporais ou espaciais perceptíveis.

As variações espaciais de temperatura, tanto do ar quanto da água, e da salinidade, dentro dos meses, podem ter sofrido interferência da alternância dos dias de coleta, horários diferentes de marés, mudanças diárias dos fatores hidrológicos e de incidência da radiação solar, além de outros fatores climáticos não avaliados (por exemplo, 
Tabela 1. Parâmetros ambientais para o período de estudo, São Caetano de Odivelas, Pará.

\begin{tabular}{|c|c|c|c|c|c|c|c|c|c|}
\hline \multirow{2}{*}{ Parâmetro } & \multicolumn{3}{|c|}{ Junho } & \multicolumn{3}{|c|}{ Julho } & \multicolumn{3}{|c|}{ Agosto } \\
\hline & P-1 & P-2 & P-3 & P-1 & P-2 & P-3 & P-1 & P-2 & P-3 \\
\hline Temperatura - $\operatorname{ar}\left({ }^{\circ} \mathrm{C}\right)$ & 29 & 27 & 32 & 31 & 34 & 31 & 30,5 & 30 & 30 \\
\hline Temperatura - água $\left({ }^{\circ} \mathrm{C}\right)$ & 28,5 & 29 & 31,5 & 29,5 & 32 & 30 & 31,5 & 29,5 & 29,5 \\
\hline $\mathrm{pH}$ & 7,1 & 7,2 & 7,5 & 7,5 & 7,5 & 7,5 & 7,1 & 7 & 7,5 \\
\hline Salinidade & 10 & 12 & 15 & 17 & 18 & 18 & 23 & 22 & 25 \\
\hline Precipitação pluviométrica (mm) & \multicolumn{3}{|c|}{352,5} & \multicolumn{3}{|c|}{280,2} & \multicolumn{3}{|c|}{81,4} \\
\hline
\end{tabular}

ventos, umidade do ar etc.). Vale ressaltar ainda que estuários constituem ambientes complexos, ecótonos entre a água doce e marinha, nos quais a interação de processos físicos, químicos, geológicos e biológicos resulta em grande variabilidade temporal e espacial, de pequena e grande escala (Pickard \& Emery, 1982).

\section{MACROFAUNA}

Neoteredo reynei foi o único Teredinidae identificado nas nove amostras coletadas, com um total de 103 indivíduos. Em cada tora, foram encontrados, em média, 11 indivíduos, sendo que junho foi o mês com menor ocorrência da espécie (média de seis indivíduos/tora) e agosto o com a maior (média de 17 indivíduos/tora). Esta espécie já havia sido registrada na costa do Pará pelos trabalhos de Andrade (1979), na Tese de Doutorado de Clara Pantoja Ferreira e por Muller \& Lana (2004), sendo considerada uma espécie comum em manguezais paraenses.

A macrofauna associada às galerias de $N$. reynei foi composta por um total de 452 indivíduos, distribuídos em 31 táxons pertencentes aos filos Nemertea (um táxon), Mollusca (três táxons), Annelida (seis táxons) e Arthropoda (21 táxons) (Tabela 2). Os grupos de invertebrados presentes no interior das galerias são principalmente marinhos (por exemplo, Nemertea, Polychaeta e Crustacea), sendo que a minoria está associada aos grupos terrestres, tais como Chilopoda e Insecta (Tabela 2). Esta composição combinada da macrofauna reflete a influência dos ambientes terrestres adjacentes no manguezal e supõe que o interior das galerias seja um local que permita a coexistência de uma grande variedade de grupos zoológicos, com distintas características ecológicas.

O número de táxons registrado foi superior aos encontrados em alguns estudos com macrofauna de substrato não consolidado em manguezais do Norte do Brasil, apesar de ser relativamente pequeno o número de amostras coletadas no presente estudo, de apenas nove. Em Bragança, Pará, Rosa Filho et al. (2006) registraram 17 táxons. No Maranhão, Oliveira \& Mochell (1999) encontraram 24 táxons. Já na ilha de Maracá, Amapá, Fernandes (2003) descreveu a ocorrência de apenas 15 táxons. Segundo Clara Pantoja Ferreira, em sua Tese de Doutorado, a diversidade da fauna encontrada nas galerias de teredos pode ser explicada pela maior estabilidade que estas oferecem, além de proteção e alimento.

A distribuição da fauna bentônica nos manguezais é influenciada, principalmente, pela grande variação da salinidade e pela disponibilidade de substrato adequado à colonização (Camargo, 1986; Duke et al., 1998). Em estuários da costa norte brasileira, em especial, as associações bentônicas de fundos moles estão submetidas a elevado estresse devido ao regime de macromarés semidiurnas e às marcantes diferenças nas taxas de precipitação ao longo do ano. Tais peculiaridades resultam em bruscas variações na salinidade, que pode variar de 0-5 (período chuvoso) a 32-35 (período menos chuvoso) (Rosa Filho et al., 2006). Consequentemente, os organismos procuram locais onde possam evitar os efeitos das oscilações do nível de maré, que modifica 
Tabela 2. Abundância total dos teredos e dos táxons associados às suas galerias, manguezal do rio Mojuim, São Caetano de Odivelas, Pará.

Continua

Teredinidae
Neoteredo reynei (Bartsch, 1920)
Macrofauna associada

Nemertea

Mollusca

Acteocina candei (Orbigny, 1842) (Gastropoda)

Detracia parana Morrison, 1951 (Gastropoda)

Melampus coffeus (Linnaeus, 1758) (Gastropoda)

Annelida

Namalycastis abiuma (Muller in Grube) 1872 (Polychaeta)

Namalycastis sp. (Polychaeta)

Namanereis pontica (Bobretzky, 1872) (Polychaeta)

Namanereis sp. (Polychaeta)

Notomastus lobatus Sasakawa, 1955 (Polychaeta)

Amphinominidae (Polychaeta)

\section{Arthropoda}

Armases angustipes Dana, 1852 (Decapoda, Crustacea)

Armases benedicti (Rathbun, 1897) (Decapoda, Crustacea)

Eurytium sp. (Decapoda, Crustacea)

Hexapanopeus paulensis Rathbun, 1930 (Decapoda, Crustacea)

Metasesarma rubripes (Rathbun, 1897) (Decapoda, Crustacea)

Pachygrapsus gracilis (Saussure, 1958) (Decapoda, Crustacea)

Uca burgersi Holthuis, 1967 (Decapoda, Crustacea)

Uca mordax (Smith, 1870) (Decapoda, Crustacea)

Uca rapax (Smith, 1870) (Decapoda, Crustacea)

Ucides cordatus (Linnaeus, 1763) (Decapoda, Crustacea)

Quadrivisio sp. (Amphipoda, Crustacea)

Gamaridae (Amphipoda, Crustacea)

Dexaminidae (Amphipoda, Crustacea)

Sphaeroma terebrans Bate, 1866 (Isopoda, Crustacea)

Dies fluminensis (Mańe-Garzón, 1944) (Isopoda, Crustacea)

Cirolana sp. (Isopoda, Crustacea)

Anobiidae (Insecta, Hexapoda)

Gryllidae (Insecta, Hexapoda)

Azteca sp. (Formicidae, Hexapoda)

\section{Continua}

Ocasião de coleta

\begin{tabular}{|c|c|c|}
\hline \multicolumn{3}{|c|}{ Ocasião de coleta } \\
\hline Junho & Julho & Agosto \\
\hline 20 & 34 & 50 \\
1 & 8 & 2 \\
\hline
\end{tabular}

2

9

5

13

8

9

4

5

2

37

2

2

16

4

2

2

1

12

4

54

3

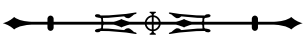


Tabela 2.

\begin{tabular}{l|c|c|c}
\hline \multicolumn{1}{c|}{ Táxon } & \multicolumn{2}{|c}{ Ocasião de coleta } \\
\cline { 2 - 4 } & Junho & \multicolumn{2}{|c}{ Julho } \\
\hline $\begin{array}{l}\text { Dolichoderus lutosus (Fr. Smith, 1858) (Formicidae, Hexapoda) } \\
\text { Lithobiomorpha (Chilopoda, Myriapoda) }\end{array}$ & 1 & 9 & 11 \\
Riqueza de táxons associados às galerias & & 27 & 22 \\
Abundância de organismos associados às galerias & 17 & 161 & 207 \\
\hline
\end{tabular}

constantemente a salinidade e mobiliza e expõe o sedimento à dessecação provocada pelo sol.

A presença de teredos possibilita a colonização dos troncos de mangue por outros grupos de organismos, uma vez que estes são os primeiros capazes de perfurálos e ocupá-los (conforme expõe Clara Pantoja Ferreira). Neoteredo reynei apresenta um tamanho considerável, podendo alcançar até $2 \mathrm{~m}$ de comprimento, construindo galerias largas no sentido das fibras da madeira (Muller \& Lana, 2004). Além da promoção de espaço explorável, segundo Turner \& Johnson (1971), os Teredinidae provêm alimento por outros invertebrados através das suas fezes e das bactérias que decompõem a madeira.

Clara Pantoja Ferreira ratifica que as toras caídas no sedimento oferecem uma variedade de microhabitats, sendo ocupadas pelos organismos desde seu córtex até as regiões mais basais, próximas às galerias de teredos. Segundo esta autora, existe uma sobreposição espacial no interior dos troncos perfurados, com a partilha de recursos entre os organismos.

A abundância dos filos que constituíram a macrofauna durante os meses de coleta encontra-se representada na Tabela 2 . Além de maior riqueza, o filo Arthropoda foi também o mais abundante, com 76,5\% dos organismos coletados, sendo que, deste total, o subfilo Crustacea representou 65,7\%. Os crustáceos com maior representatividade foram os Decapoda $(38,9 \%$ do total de organismos) e os Isopoda $(22,8 \%$ do total de organismos). O grupo Crustacea também apresentou as espécies mais abundantes (Armases angustipes Dana, 1852, Uca burguesi Holthuis, 1967 e Cirolana sp.) (Tabela 2).

A sequência de representatividade dos demais grupos taxonômicos foi a seguinte: Annelida Polychaeta (16\% do total de indivíduos); Mollusca Gastropoda (10,8\% do total de indivíduos) e Nemertea (2,4\% do total de indivíduos). A composição e o padrão de ocorrência dos táxons da macrofauna - maior abundância e riqueza para Decapoda, Polychaeta e Gastropoda - são semelhantes aos registrados por Clara Pantoja Ferreira. Em estuários tropicais, comumente, a macrofauna bentônica é dominada por Crustacea, Mollusca e Annelida (Gambi et al., 1997; Little, 2000) e os decápodes geralmente apresentaram o maior número de espécies nas comunidades de manguezais tropicais (Miranda et al., 1988). Sobre este último grupo, o número de espécies aqui registrado (dez) é similar ao encontrado pelo estudo de Sousa et al. (1998) com troncos caídos de várias espécies de mangues em manguezais de Alagoas (11), evidenciando que os decápodes utilizam habitualmente as árvores de mangue como locais de abrigo. A maior parte dos decápodes aqui coletados era composta por juvenis e fêmeas ovígeras, o que ressalva a importância das toras como locais de proteção.

No decorrer dos meses de estudo, houve um aumento na abundância e no número de táxons da macrofauna, sobretudo para os grupos tipicamente marinhos e estuarinos, como anelídeos e crustáceos (Tabela 2). Tais incrementos se refletiram nos descritores biológicos (Figura 1), embora não tenha sido observada diferença 


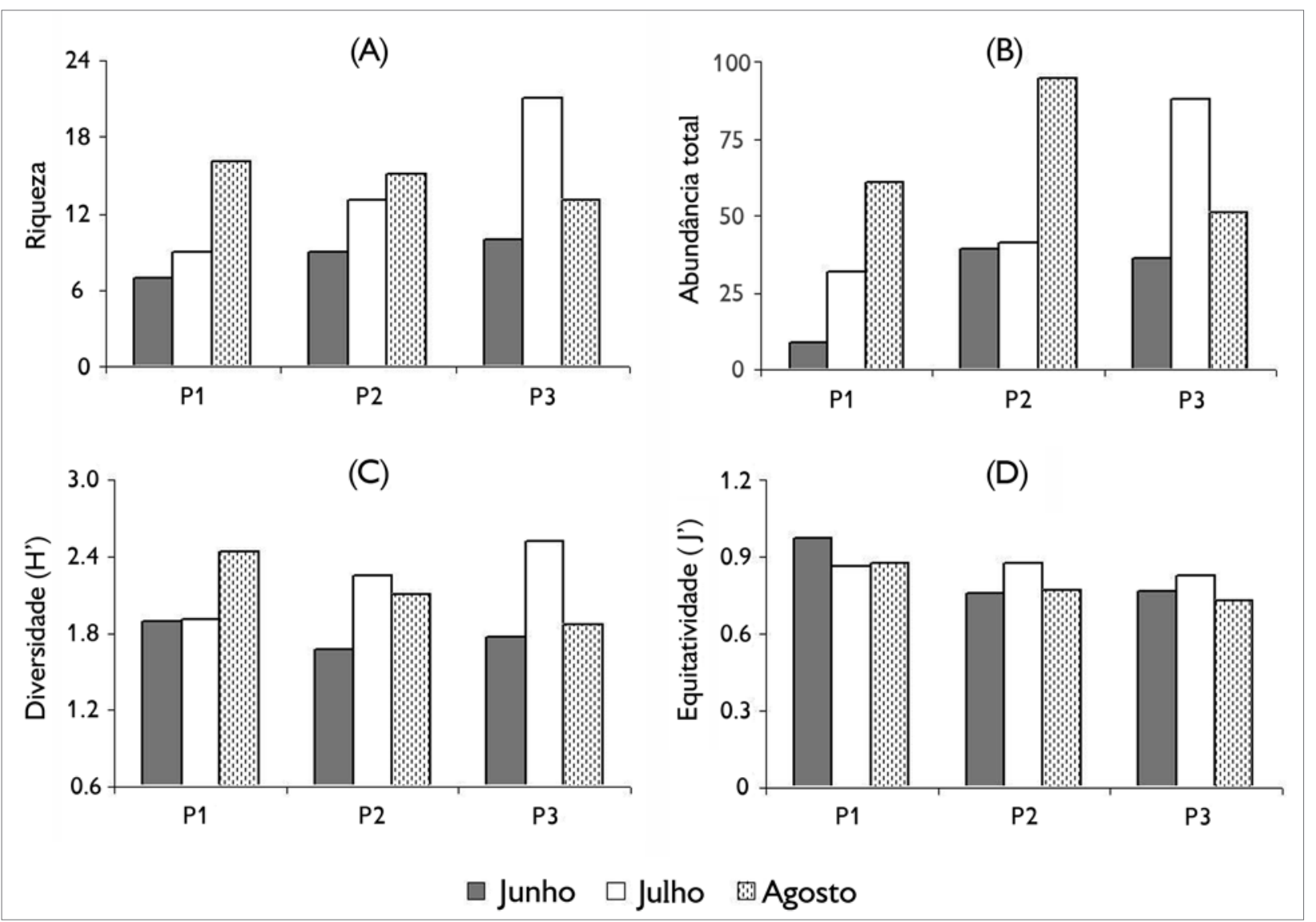

Figura 1. Riqueza (A), abundância (B), diversidade (C) e equitatividade (D) da macrofauna durante o período de amostragem. Manguezal do rio Mojuim, São Caetano de Odivelas, Pará.

estatística significativa destes entre os meses (valores de $p$ $>0,05)$. Vale lembrar que o pequeno número de amostras para cada mês, três apenas, pode ser insuficiente para se obter resultados estatísticos mais relevantes e seguros.

$\bigcirc$ aumento gradual da abundância e diversidade da fauna associada, assim como o de ocorrência dos teredos, seguiu a diminuição da pluviosidade e aumento da salinidade no estuário (Tabela 1). $\bigcirc$ mês de junho na região Norte pode ser considerado como de transição entre o período chuvoso e menos chuvoso e durante o qual o volume de chuvas ainda é relativamente elevado (Moraes et al., 2005). A baixa salinidade observada neste mês e nos antecedentes do período chuvoso pode ter atuado como uma barreira fisiológica para as espécies estuarinas e marinhas (Kenish, 1986) que melhor se estabeleceriam nas ocasiões menos chuvosas e com valores de salinidade mais elevados. Sobre os teredinídeos, sabe-se que um fator limitador preponderante da sua distribuição em águas estuarinas é a tolerância das suas larvas à baixa salinidade durante o assentamento e metamorfose. A maioria das espécies tem um melhor recrutamento em salinidades acima de 20 (Rayner, 1979) e valores entre dez e 14 são críticos para a sobrevivência mesmo de indivíduos já estabelecidos (Barreto et al., 2000).

Neste sentido, tanto os teredos quanto a fauna associada às suas galerias devem ter um padrão de distribuição temporal, influenciado pelas variações de 
precipitação pluviométrica e de salinidade, porém tal fato deve ser confirmado em estudos sazonais.

Os valores de riqueza, abundância e diversidade registrados durante os dois primeiros meses de coleta foram menores no sítio P1 (Figura 1), aumentando em P2 e P3. A ausência de réplicas biológicas dentro de cada sítio, assim como a análise de mais parâmetros ambientais, impossibilita comparações e conclusões seguras. Contudo, vale lembrar que os menores valores de salinidade registrados em P1, assim como o fato deste ser o sítio mais próximo de uma área urbana, com maior influência dos impactos antrópicos (por exemplo, atividade portuária, desmatamento, esgoto e lixo urbano), podem ter contribuído para tais registros. Já P2 e P3 apresentaram maiores valores de salinidade e estão mais próximos de um canal de maré, o que pode contribuir para maior inundação destes sítios, maior imersão e menor tempo de exposição das toras ao ar e irradiação solar, bem como a manutenção e transporte de organismos para o interior das mesmas.

\section{CONCLUSÕES}

A macrofauna associada às galerias construídas por teredos foi constituída majoritariamente por grupos tipicamente estuarinos, comuns em manguezais tropicais. Levando em consideração o pequeno número amostral, a pontualidade temporal e espacial e os resultados obtidos pelo presente estudo, pode-se considerar como sendo abundante e rica a fauna que ocupa este habitat.

O interior dos troncos de mangue é um local favorável ao estabelecimento dos organismos, provavelmente por: 1) proporcionar estrutura física estável, sofrendo menos influência de correntes e oscilações dos níveis de maré e da salinidade; 2) oferecer proteção contra predadores e a dessecação provocada pelo sol; 3) apresentar um grande número de microhabitats; 4) oferecer recurso alimentar; e 5) possibilitar a inclusão de grupos de invertebrados terrestres menos tolerantes às condições salinas.

Os resultados obtidos constituem uma contribuição ao conhecimento da fauna associada às galerias de teredos, sendo necessários estudos mais apurados, tanto temporal quanto espacialmente, que possam traduzir aspectos mais amplos sobre sua ecologia.

\section{REFERÊNCIAS}

ANDRADE, J., 1979. Folclore na região do Salgado, Pará. Teredos na alimentação; profissões ribeirinhas: 1-83. Escola de Folclore, São Paulo.

BARRETO, C. C., A. O. R. JUNQUEIRA \& S. H. G. SILVA, 2000. The effect of low salinity on teredinids. Brazilian Archives of Biology and Technology 43(4): 399-407.

BRANCH, G. M. \& A. PRINGLE, 1987. The impact of the send prawn Callianassa kraussi Stebbing on sediment turnover and on bacteria, meiofauna, and benthic microflora. Journal of Experimental Marine Biology and Ecology 107(3): 219-235.

CAMARGO, T. M., 1986. Fauna do manguezal. In: Y. SCHAEFFERNOVELLI \& G. CINTRON: Guia para estudo de áreas de manguezal: sua estrutura, função e flora: 32-40. Caribbean Ecological Research, São Paulo.

DUKE, N. C., M. C. BALL \&J. C. ELLISON, 1998. Factors influencing biodiversity and distributional gradients in mangroves. Global Ecology and Biogeography Letters 7(1): 27-47.

FERNANDES, M. E. B., 2003. Macroendofauna bêntica de substrato móvel. In: M. E. B. FERNANDES (Org.): Os manguezais da costa norte brasileira: 87-104. Fundação Rio Bacanga, São Luís.

FRITH, D., 1976. Animals associated with sponges at North Hayling Hampshire. Zoological Journal of the Linnean Society 58: 353-362.

GAMBI, M. C., G. CONTI \& C. S. BREMEC, 1997. Polychaete distribution, diversity and seasonality related to seagrass cover in shallow soft-bottoms of the Tyrrenian Sea (Italy). Scientia Marina 26(1-2): 1-17.

KENISH, M. J., 1986. Ecology of estuaries: biological aspects: 2: 1-391. CRC Press, Florida.

LEVINTON, J. S., 2001. Marine biology: function, biodiversity, ecology: 1-420. Oxford University Press, Oxford.

LITTLE, C., 2000. The biology of soft shores and estuaries: 1-252. Oxford University Press, Oxford.

MARTORANO, L. G., 1993. Estudos climáticos do Estado do Pará: Classificação climática de Köppen e deficiência hídrica: 1-53. SUDAM/EMBRAPA/SNLCS, Belém.

MiRANDA, P. T. C., F. F. G. GURGEL, M. A. F. LIBERATO, M. T. OLIVEIRA \& T. L. B. ARRUDA, 1988. Comunidades bentônicas em raízes de Rhizophora mangle Linnaeus, no manguezal do Rio Ceará (Ceará-Brasil). Arquivos de Ciências do Mar 27: 101-110. 
MORAES, B. C., J. M. N. COSTA, A. C. L. COSTA \& M. H. COSTA, 2005. Variação espacial e temporal da precipitação no Estado do Pará. Acta Amazônica 35(2): 207-214.

MULLER, A. C. P. \& P. C. LANA, 2004. Manual de identificação de moluscos bivalves da família dos teredinídeos encontrados no litoral brasileiro: 1-148. Editora da UFPR, Curitiba.

OLIVEIRA, M. \& F. R. MOCHEL, 1999. Macrofauna bêntica de substratos móveis de um manguezal sob impacto das atividades humanas no sudoeste da Ilha de São Luiz, Maranhão, Brasil. Boletim do Laboratório de Hidrobiologia 12: 75-93.

PICKARD, G. L. \& W. J. EMERY, 1982. Descriptive physical oceanography: an introduction: (4): 1-210. Pergamon Press, New York.

PROST, M. T., A. C. MENDES, J. F. FAURE, J. F. BERREDO, M. E. C. SALES, L. G. FURTADO, M. G. S. SILVA, C. A. SILVA, I. NASCIMENTO, I. GORAYEB, M. F. V. SECCO \& L. M. LUZ, 2001. Manguezais e estuários da costa paraense: exemplo de estudo multidisciplinar integrado (Marapanim e São Caetano de Odivelas). In: M. T. PROST \& A. C. MENDES: Ecossistemas Costeiros: Impactos e Gestão Ambiental: 75-87. Museu Paraense Emílio Goeldi, Belém.

RAYNER, S. M., 1979. Comparison of the salinity range tolerated by Teredinids (Mollusca: Teredinidae) under controlled conditions with that observed in an estuary in Papua New Guinea. Australian Journal of Marine and Freshwater Research 30(3): 521-533.

RIMMER, M. A., S. L. BATTAGLENE \& P. L. DOSTINE, 1983. Observation of Bankia australis Calman (Mollusca: Teredinidae) in the Patonga Creeck mangrove swamp, New South Wales. Australian Journal of Marine and Freshwater Research 30(2): 355-357.

ROSA FILHO, J. S., D. V. BUSMAN, A. P. VIANA, A. M. GREGÓRIO \& D. M. OLIVEIRA, 2006. Macrofauna bentônica de zonas entremarés não vegetadas do estuário do rio Caeté (Bragança-PA). Boletim do Museu Paraense Emílio Goeldi. Ciências Naturais 1(3): 85-96.
SANTOS, C. L. T., D. S. TAVARES \& C. A. ROCHA-BARREIRA, 2007. Invertebrados associados a galerias de teredos (Mollusca, Bivalvia) em troncos de Rhizophora mangle e Avicennia schaueriana no estuário do rio Pacoti, Ceará, Brasil. In: XX Encontro Brasileiro de Malacologia, Livro de resumos: 314-314. XX EBRAM 2007, Rio de Janeiro.

SEPÚlVEDA, R. D., R. MORENO \& F. D. CARRASCO, 2003. Diversidad de macroinvertebrados asociados a arrecifes de Phragmatopoma moerchi Kinberg, 1867 (Polychaeta: Sabellariidae) en el intermareal rocoso de Cocholgüe Chile. Gayana 67(1): 45-54.

SOUSA, E. C., T. C. S. CALADO \& F. D. GUIMARÃES, 1998. Aspectos ecológicos dos Crustacea Decapoda habitantes de troncos no complexo estuarino-lagunar Mundaú/ManguabaAlagoas. Trabalhos de Oceanografia da Universidade Federal de Pernambuco 26(1): 117-122.

THIEL, M. \& N. ULLRICH, 2002. Hard rock versus soft bottom: the fauna associated with intertidal mussel beds on hard bottoms along the coast of Chile, and considerations on the functional role of mussel beds. Helgoland Marine Research 56(1): 21-30.

TURNER, R. D., 1966. A survey and illustrated catalogue of the Teredinidae: 1-265. Museum of Comparative Zoology, Harvard University, Cambridge.

TURNER, R. D. \& A. C. JOHNSON, 1971. Biology of marine wood-boring mollusks. In: E. B. G. JONES \& S. K. ELTRINGHAM (Eds.): Marine borers, fungi and fouling organisms of wood: 31-45. Organization for Economic Co-operation and Development, Paris.

YOUNG, P. S., 1986. Qualitative and quantitative analysis of the fauna associated to hermatypic corals (Coelenterata, Scleractinia) on reefs of Joao Pessoa, Paraíba (Brazil). Revista Brasileira de Biologia 46(2): 99-126.

Recebido: 12/09/2008 Aprovado: 23/04/2009

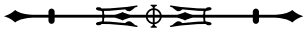


